\title{
Quality Improvement Initiative: Foley Catheters in Patients Undergoing Pectus Deformity Repair
}

\author{
Jordan Crabtree ${ }^{1}$, Troy Markel ${ }^{2}$
}

${ }^{1}$ Indiana University School of Medicine; ${ }^{2}$ Indiana University School of Medicine, Department of Surgery

\section{Background and Hypothesis:}

Pectus excavatum and carinatum are sternal deformities that often require surgeries with significant postoperative pain that can limit a patient's ability to void. An indwelling urinary catheter is placed for the operation, but is often removed on the first postoperative day due to concerns of infection, which begs the necessity of these catheters in the first place. In this study, we hypothesized that there would be no increased risk of urinary retention or urinary tract infection irrespective of whether urinary catheters were utilized.

\section{Experimental Design:}

A pre/post quality improvement study was undertaken of patients undergoing pectus excavatum/carinatum repair between June 1, 2015 and May 31, 2022. The pre intervention group spanned from June 1, 2015 to May 31, 2021, where Foley catheters were placed intraoperatively and removed the first postoperative day. Pediatric surgery and anesthesia groups changed practice effective June 1, 2021 and decided to no longer utilize urinary catheters in pectus repairs. Pre-intervention patients were acquired through the surgery billing database. Post-intervention patients were acquired prospectively. Rates of urinary retention (any event requiring mechanical intervention for voiding) and urinary tract infections (UTI, $>100,000$ CFU bacteria/mL urine) were compiled into REDCap. Data are expressed as percent of the total cohort.

\section{Results:}

Of 179 patients undergoing pectus repair (162 Excavatum, 17 Carinatum), 12 patients (6.7\%) in the pre-intervention group experienced urinary retention. Of these, 9 received in/out catheterization, 1 had a Foley catheter replaced, and 2 underwent in/out catheterization and had a Foley replaced. There were no urinary tract infections recorded among the pre-intervention group.

\section{Conclusion and Potential Impacts:}


Urinary retention and UTI are rare in patients who have an indwelling urinary catheter. Data acquired over the next year from the post-intervention group will help determine the true necessity for these catheters in the perioperative pectus population. 